\title{
25 \\ Simulation of wires handling for the automation of industrial processes
}

P. Denti, P. Dragoni, G. Frugoli, and C. Rizzi

KAEMaRT Group, Department of Industrial Engineering University of Parma,

Viale delle Scienze, 43100 Parma, Italy

Tel. +39521905701, Fax. +39521905705

E-mail: [denti, frugoli, rizzi]@ied.eng.unipr.it

\begin{abstract}
This paper considers the automation of industrial processes; in particular we focus attention on those operations that require the manipulation of wires and cables, such as laying.

To this end, we used a software prototype, named SoftWorld, that provides a graphical environment to simulate the handling machinery integrated with the dynamic and static behavior of the material.

To fulfill our goal we proceeded in three steps: analysis of possible wire models, identification of possible approaches to simulate the handling process, and validation through experimental tests.

In order to cover most of the typical problems in wire simulation, different models have been considered to describe wires and simulate their behavior. The objective has been to investigate different approaches analyzing advantages and disadvantages with reference to the goal of the simulation.

In the same way, for the simulation of the handling process we considered different approaches that imply different computational costs and give different levels of accuracy.

Results obtained will be described as well as final considerations and future developments.
\end{abstract}

Keywords

Wire handling, nonrigid materials modeling and simulation, automation. 


\section{INTRODUCTION}

Mainly in the automotive and aerospace sectors, wire and flexible tube laying design is getting more and more important. Several sensors and actuators are used even in low-cost vehicles both for safety and performance. Examples include diagnotic systems, ABSs, climate control systems, airbags, integrated hi-fi systems, and so on.

The automation of wire laying processes is usually considered as a 'geometrical' planning problem but, in general, this is not correct: several 'physical' parameters should be taken into account. Wires and flexible tubes in vehicles are exposed to damages due to high stress, high temperature, and vibration. Some of the damage causes could be eliminated during the design phase by taking into account the behavior of the vehicle under operating conditions. Tools for off-line analysis could be very effective in detecting design pitfalls.

In such a context, we developed a software prototype, named SoftWorld, that provides a graphical environment to simulate the handling machinery integrated with the dynamic and static behavior of the material. At first the system was experimented with considering typical operations in the clothing industry, e.g. pick and place of garment pieces or garment draping on digital mannequins (Cugini 1994, Denti 1996). Now we focus our attention on a different class of flexible objects, wires and cables, and on processes that require their manipulation, e.g., vehicle assembly or recycling.

Wire behavior simulation can be carried out at different levels of accuracy (and computational cost) depending on problem requirements. In the following, different approaches to model wires are shown with their advantages and disadvantages, in order to cover most of the typical problems in wire simulation.

Tests carried out to compare the potential of different wire models and approaches for process simulation will be described as well as final considerations on the results obtained.

\section{NONRIGID MATERIALS REPRESENTATION}

The approach adopted in SoftWorld (Denti, 1996) to model flexible materials is based on the known particle-based approach (Witkin, 1995, Volino, 1995, Eberhardt, 1996): with a particle-based representation, a deformable object is described as a set of mechanical elements (particles) and forces acting among them, representing the macro-behavior of the material (e.g., elasticity, plasticity). For example, a piece of fabric can be modeled as a grid of particles with a distribution of forces, like bending, stretching and repelling. Different materials are modeled using different:

- particle meshes;

- particle masses;

- sets of inter-particle forces. 
In order to simulate a manufacturing process, it is not sufficient to model only the deformable objects but it is necessary to consider external forces that could be applied to the objects (e.g., the action of a gripper), constraints that must be respected (e.g., a predefined trajectory), and rigid obstacles (e.g., a worktable).

SoftWorld permits modeling not only different classes of flexible objects but also the physical environment surrounding them, in terms of external forces, constraints and rigid obstacles. Figure 1 portrays the high-level architecture of the prototype. Two main components can be identified: SoftLand, the modeling and simulation library, and SoftInterface, the graphical user interface.

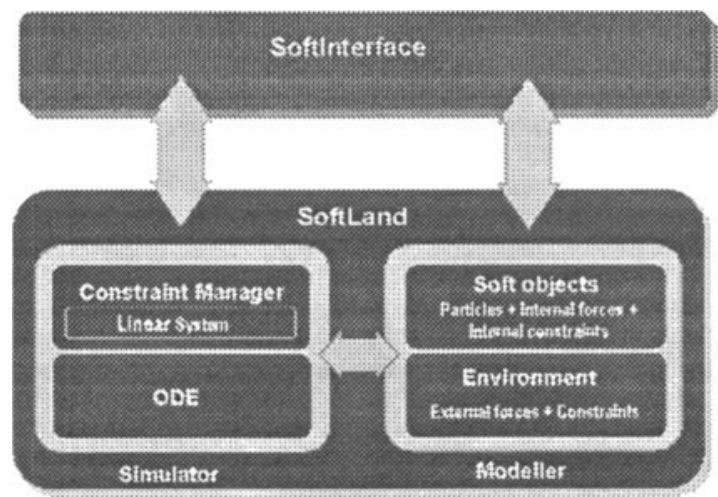

Figure 1 High-level architecture of SoftWorld

SoftLand can be subdivided in two main modules: the modeler and the simulator. The modeler comprehends all the necessary functionalities for the definition of nonrigid objects and surrounding environment. As already stated, nonrigid objects are represented by a set of particles interconnected by internal forces and subjected to internal constraints, while the environment is a set of external forces and constraints. The simulator comprehends:

- an ODE (Ordinary Differential Equation) numerical solver;

- a Constraint Manager, whose aim is to manage constraints and obstacles during the simulation.

The system also provides the end-user with a graphical environment, SoftInterface, to model nonrigid materials and to simulate their behavior during handling processes. Through the user interface, the system allows a designer to:

- describe nonrigid objects together with their physical properties as a set of particles interconnected by internal forces (describing the physical behavior) and subjected to internal constraints;

- describe a virtual physical environment as a set of external forces (e.g., gravity), rigid obstacles, and constraints;

- simulate the behavior of the objects in the defined virtual physical environment, producing both numerical and graphical output; 
- produce high-quality graphical outputs using different rendering techniques. Up to now the prototype has been used:

- to model and simulate flexible industrial products, in this case wires;

- to simulate automated handling processes.

In the following, we illustrate different representations that can be adopted to model and simulate the behavior of wires during handling operations.

\section{WIRES REPRESENTATION AND SIMULATION}

To simulate wire handling two problems have to be considered:

- how to represent wires (in our case, how to do it using a particle-based model);

- how to describe the interaction with the environment in terms of external forces, constraints and obstacles.

The particle-based approach can be seen as a macro-molecular representation. The accuracy level needed for the simulation determines the particle mesh resolution of the model. Tending toward the exact molecular representation, both for particles and forces, we obtain simulation results that better approximate the real behavior. Of course, the exact molecular representation is too complicated to handle and, moreover, approximated solutions are in general good enough for common purposes; different levels of approximation are usually required for different problems.

Our objective has been to investigate different models for wire behavior simulation and find out which is the best suitable with reference to the goal of the simulation.

Different representations have been considered in order to describe wires and simulate their behavior:

- unidimensional;

- surface-like;

- solid-like.

Each model is characterized by a different level of realism and computational cost.

Even for the problem of the interactions with the environment, considerations can be done taking into account the level of realism required. Several parameters can be considered or ignored: in a simulation of a grasping operation we could take into account the roughness of the gripper fingers, the force exerted by the gripper (and therefore the deformation generated), and collisions with other parts of the robot arm.

Two approaches, corresponding to different problems, have been identified:

- imposing a trajectory constraint;

- considering the real gripper action. 
In order to verify and evaluate the different models and simulation approaches, two tests have been carried out: simulation of automatic wire laying and simulation of wire deformation due to the action of a gripper.

In the following, wire models and simulation approaches will be described with reference to the tests considered.

The unidimensional model is the simplest representation of a wire or a cable, by which the object is represented as a string of particles, with stretching, repelling and bending forces that connect each particle to its previous and next neighbors. Due to the fact that only one of the three dimensions of the object is considered, this model is suitable for simulations in which computational speed is more important than realism of the object behavior or when the study of transversal deformations is not relevant. Therefore this model is not satisfactory enough for our tests.

\section{SIMULATION OF AUTOMATIC WIRE LAYING}

This test considers the simulation of wire laying operations: we suppose to grasp a wire and move it along a specified trajectory. Various obstacles represent the surrounding environment. Therefore, in this case the focus is on the behavior of the wire during the operation.

The surface-like model has been adopted to represent the wire. The object is modeled with a cylindrical surface: the process of discretization is carried out by positioning the particles on the intersection of the isoparametric curves of the surface (Figure 2). In this way, the model becomes a series of connected rings of particles distributed along the central axis of the object.

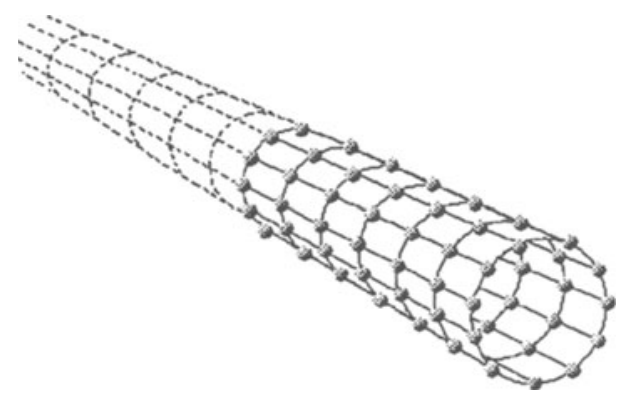

Figure 2 Wire represented by a set of particles distributed on a cylindrical surface

Between every pair of rings we set elastic forces simulating the resistance to pulling and compressive stress. Other forces are also set in order to simulate the bending resistance and the cross-section deformation tension.

This model offers a good level of realism and acceptable computational cost. With respect to the unidimensional model, it implies more complex collision detection due to a more complex geometry and a bigger set of parameters to be 
tuned in order to obtain the real behavior. However, this approach offers an average level of realism at an average computational cost and the possibility to take into account the torsional stress.

The simulation is carried out by imposing a trajectory constraint. This approach is based on the following assumptions:

- a grasped object, if the operation is correctly done, can be considered forming a single unit with the gripper;

- material deformations generated by the gripper action can be ignored.

The trajectory constraint has been considered in order to impose a particular motion law to some particles. In order to define the constraint, we first determine the area involved in the grasping operation and then the particles contained within this area are constrained to follow the gripper trajectory. In such a way, the grasping action is simulated imposing a predefined trajectory to the particles detected.

Main characteristics of this approach are:

- higher simulation speed, if compared with the other approach that will be described in the following;

- independence from the shape of the contact surface between object and gripper and from the friction coefficient;

- low level of realism, because the grasping action on the material is not simulated.

The layout used for the test is as follows (Figure 3):

- a wire laying on a table;

- a gripper that holds one end of the wire;

- obstacles represented by thin walls.
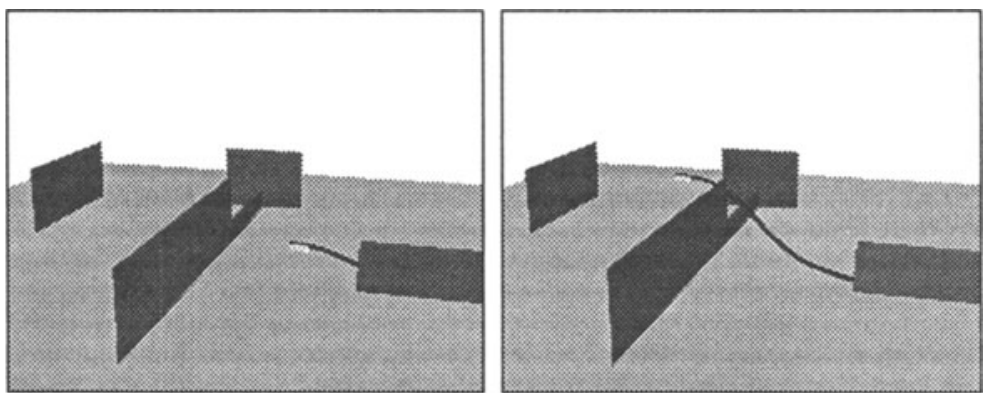

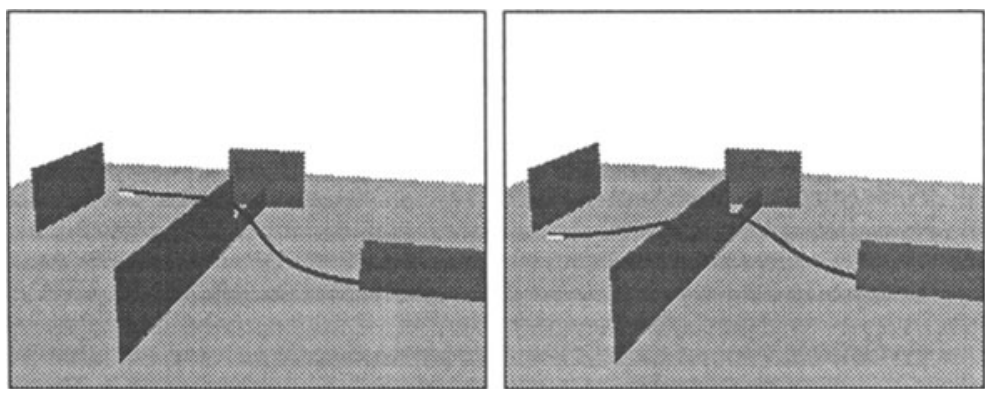

Figure 3 Wire laying simulation

The wire is grasped at one of its ends and moved along a predefined trajectory. The object is therefore constrained to follow the trajectory imposed by the gripper. Figure 3 shows some steps of the behavior of a wire when manipulated as described. In the sequence shown in Figure 3, it is possible to see the behavior of the wire when colliding with obstacles.

\section{SIMULATION OF THE ACTION OF A GRIPPER}

The second test considers the simulation of the action of a robot gripper. The most important aspect of this example is the deformation of the material due to the external forces applied.

A three-dimensional model of the wire is more suitable for this simulation since it offers a higher level of realism. A possible discretization can be the decomposition of the object into small cubes (in the same way as if we were using a voxel representation), which will be represented by particles (Figure 4a). Generality is the main characteristic of this type of discretization, in fact this model could be adopted for any shape, not only for cylinder-like objects.

However, we loose axial symmetry, and a purely homogeneous discretization may cause large irregularities of the object surface (especially when using a small number of particles).

A more suitable discretization for a cylindrical object such as a wire is to displace the particles radially within the cross-section as shown in Figure 4b: in this way the set of inter-particles forces is arranged in a symmetric way within the section.

(a)

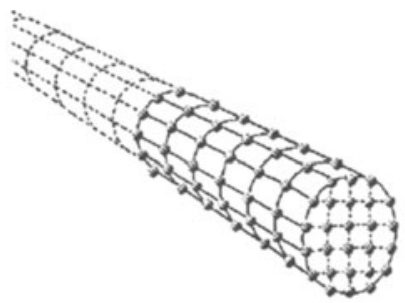

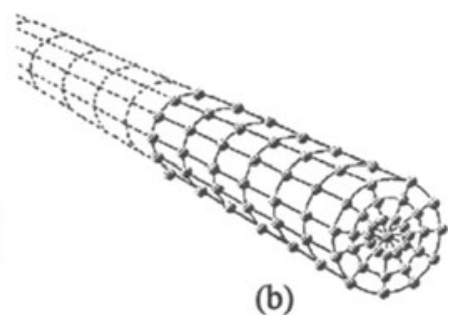

(b)

Figure 4 3D representation of the wire: (a) cubic-based mesh, (b) radial-based mesh 
With respect to the previous model, this one implies:

- higher level of realism, because all the three dimensions of the object are considered;

- bigger number of particles and forces, that implies higher computational cost.

The gripper is represented as a set of rigid objects whose surfaces are characterized by a roughness value. The rigid objects defining the gripper must follow a given trajectory in the space and when they touch the wire, a mechanical action of compression and friction on the wire surface is accomplished.

With respect to the previous approach, we have:

- higher level of realism: in this case we take into account the friction between the wire and the rigid objects as well as the wire deformation;

- possibility to calculate the force and the medium/maximum momentum of the gripper during a time interval: this can be useful to properly quantify the mechanical characteristics of the gripper;

- generality: we can define a gripper or some other mobile rigid object that will interact with a generic nonrigid object in a proper way;

- higher computational cost: this approach implies complex collision detection between the gripper and the wire and the calculation of friction and compression;

- the simulation requires a model characterized by a high level of physical realism, otherwise the simulation can produce completely wrong results.

The end-effector of the gripper is modeled by two flat plates. The wire lays on a worktable and the gripper grasps and then translates it. The simulated action is simpler than the previous but the models of the objects present in the scene are more complex. Figure 5 shows the behavior of the wire when manipulated as described: the deformation due to the action of the gripper can be seen. 


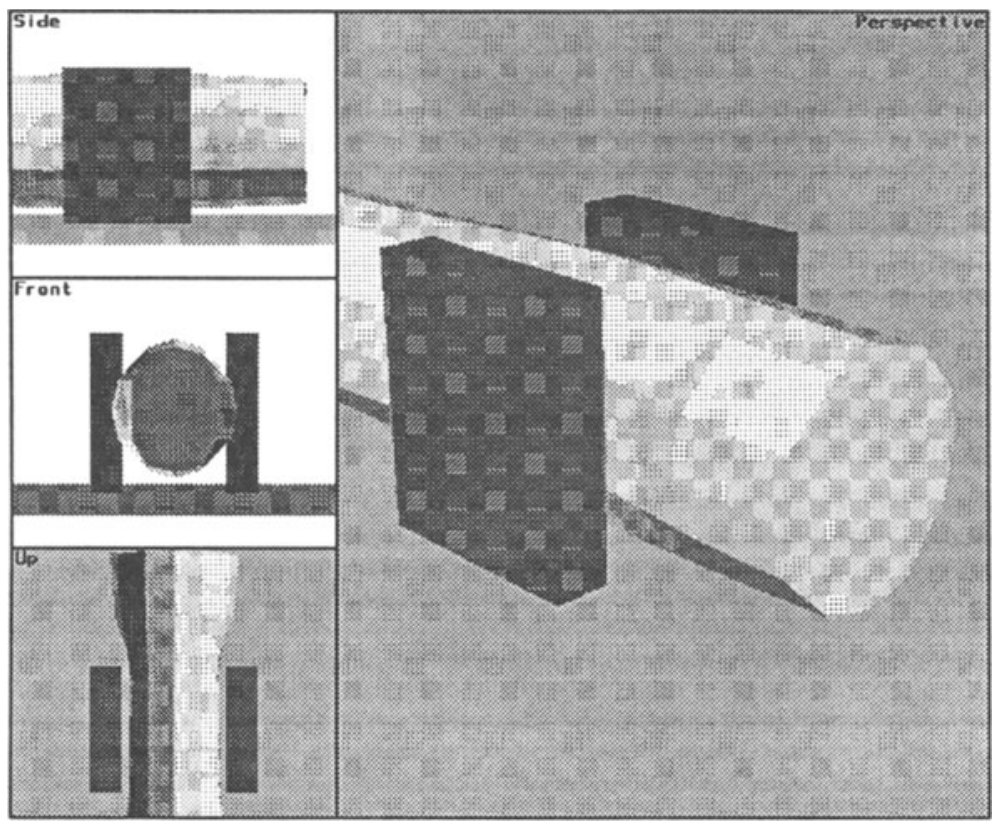

Figure 5 Simulation of the action of the gripper

\section{CONSIDERATIONS}

In some cases, it is convenient to adopt a combination of the described models. For example, in order to simulate a real electric cable, we can model the external shell as a cylindrical surface and use any of the other approaches to model the core. We can obtain different levels of realism and computational cost by using different combinations.

Even for the problem of the interaction it can be useful to adopt a mixed approach that is a combination of the two approaches described. It consists of two steps: at first we calculate the wire deformation, due to the gripper action, following the second approach; then, supposing the wire is fixed with respect to the gripper (that means it cannot slip away) and the deformation constant, we follow the first approach.

This technique is based on the following approximation: the pressure exerted by the gripper deforms the material only when it grasps it. Any further movement of the gripper does not change the effect of the finger pressure. This assumption is quite reasonable in several cases and it allows lowering significantly the computational cost of the second approach described.

Main characteristics are:

- possibility to take into account, even if partially, the wire deformation;

- average computational cost during the second phase of the simulation;

- more complex algorithm, because we must perform it in two different steps.

This mixed approach is currently under development. 


\section{CONCLUSIONS}

We showed how wires and cables can be modeled and simulated using a particlebased approach. Analyzing the techniques studied, we can see that the particlebased representation exhibits a high level of generality and flexibility. The basic idea of discretizing the object into a set of particles does not limit very much the set of simulation techniques applicable. Several algorithms, very different among themselves, can be easily tested; even different levels of precision can be obtained using the same basic modeling idea. All the modeling and simulation approaches described here are based on the same software package (SoftWorld), but many other approaches to the wire manipulation problem can be tested.

\section{REFERENCES}

Cugini, U. and Denti, P. and Ippolito, M. and Rizzi, C. (1994) Design and simulation of non-rigid materials handling systems, in Proceedings of Euriscon 94, III, 1424-1432, Malaga, Spain.

Denti, P. and Dragoni, P. and Frugoli, G. and Rizzi, C. (1996) SoftWorld: a system to simulate flexible products behaviour in industrial processes, in Proceedings of ESS 1996, II, 235-239, Genova, Italy.

Eberhardt, B., Weber, A., and Strasser, W. (1996) A fast, flexible, particle-system model for cloth draping, in IEEE Computer Graphics and Applications, 16, 5, 52-59.

Volino, P., Courchesne, M. and Magnenat Thalmann, N. (1995) Versatile and efficient techniques for simulating cloth and other deformable objects, in Proceedings of SIGGRAPH 95, Los Angeles, California, USA.

Witkin, A. et al. (1995) Physically based modeling, in SIGGRAPH 95 Course Notes, 34, Los Angeles, California, USA.

\section{ACKNOWLEDGEMENTS}

The authors would like to thank the EU Commission and all partners of SKILLMART project for their invaluable support in this research.

\section{BIOGRAPHY}

Paolo Denti is currently a post-doc fellow at the University of Parma, Department of Industrial Engineering. He received the Laurea Degree in Computer Science from University of Milan in 1991. His research interests include physically-based modeling and simulation, motion planning in robotics, computational geometry, and augmented reality.

Paolo Dragoni is currently a fellow at the University of Parma, Department of Industrial Engineering. He received the Laurea Degree in Electronic Engineering from Politecnico di Milano in 1995. Since 1995 he has been working in the field of modeling and simulation of nonrigid materials behavior for industrial applications. Current research interests include 
physically-based modeling and simulation, computational geometry, object-oriented programming.

Giancarlo Frugoli is currently a Ph.D. student at the University of Parma, Department of Industrial Engineering. He received the Laurea Degree in Electronic Engineering from Politecnico di Milano in 1996. Since 1995 he has been working in the field of modeling and simulation of nonrigid materials behavior for industrial applications. Current research interests include physically-based modeling and simulation, human-computer interaction, object-oriented programming.

Caterina Rizzi is currently Associate Professor of Computer Aided Design at the University of Parma, Faculty of Engineering. She received the Laurea Degree in Physics from University of Milan in 1985. She has been working in the area of CAD, CAM and CIM since 1985 with particular interest in the following topics: solid modeling, parametric surfaces, man-machine interaction in industrial applications, user interface development tools, nonrigid materials modeling and simulation, off-line programming. 